\title{
A Comparison of Comorbidity and Psychological Outcomes in Children and Adolescents with Attention-Deficit/ Hyperactivity Disorder
}

\author{
Yoon Huh", Inchul Choi ${ }^{1}$, Misun Song ${ }^{1}$, Sunyoung Kim², Sungdo David Hong ${ }^{1}$ and Yoosook Joung ${ }^{\bowtie}$ \\ ${ }^{1}$ Department of Psychiatry, Samsung Medical Center, Sungkyunkwan University School of Medicine, Seoul, Korea \\ ${ }^{2}$ Department of Psychiatry \& Behavioral Science, Ajou Universiy School of Medicine, Suwon, Korea
}

\begin{abstract}
Objective The purpose of this study was to compare psychiatric comorbid disorders and psychological outcomes in children and adolescents with Attention-deficit/hyperactivity disorder (ADHD).

Methods Subjects were divided into a child group (aged under 12 years) and an adolescent group (aged 12 years and above). All subjects were diagnosed with ADHD based on the DSM IV diagnostic criteria using the Kiddie-Schedule for Affective Disorders and Schizophrenia-Present and Lifetime Korean Version (K-SADS-PL-K). The K-SADS-PL-K was also used to evaluate those psychiatric disorders comorbid with ADHD. And the Korean version of the Child Behavior Checklist (K-CBCL) was used to examine the subjects' psychological outcomes.

Results The rate of comorbidity in adolescent group was significantly higher than that in the child group. In particular, the adolescent group had a significantly higher ratio of comorbid conduct disorder and mood disorder than the child group. With respect to the predominantly inattentive type and Not Otherwise Specified, the school subscale scores on the K-CBCL for the children were significantly higher than those for the adolescents.

Conclusion These results suggest that the psychiatric comorbidity may differ between adolescents and children with ADHD. Therefore when treating adolescents with ADHD, more careful assessment and treatment targeting a range of comorbidities are needed.
\end{abstract}

Psychiatry Investig 2011;8:95-101

Key Words Children, Adolescent, Comorbidity, ADHD.

\section{INTRODUCTION}

Attention-deficit/hyperactivity disorder (ADHD) is one of the most common psychiatric disorders of childhood and adolescence and is characterized by symptoms of inattention, hyperactivity and impulsivity. Worldwide, it occurs in $5.3 \%$ of school-aged children. ${ }^{1}$

Inattention is an impairment of cognitive function, whereas hyperactivity and impulsivity are impairments of behavioral function, both of which accompany various other psychiatric

Received: May 30, 2010 Revised: December 23, 2010

Accepted: January 15, 2011 Available online: February 20, 2011

$\triangle$ Correspondence: Yoosook Joung, MD, PhD

Department of Psychiatry, Samsung Medical Center, Sungkyunkwan University School of Medicine, 50 Irwon-dong, Gangnam-gu, Seoul 135-710, Korea Tel: +82-2-3410-3589, Fax: +82-2-3410-0050, E-mail: yschoung@skku.edu

(a) This is an Open Access article distributed under the terms of the Creative Commons Attribution Non-Commercial License (http://creativecommons.org/licenses/bync/3.0) which permits unrestricted non-commercial use, distribution, and reproduction in any medium, provided the original work is properly cited. problems. Such psychiatric problems can create control problems within the family and throughout school life. Some of the effects of ADHD include poor academic performance, learning disorders, aggressive behavior, temper tantrums or social isolation, and alienation due to frequent peer conflict. As this disorder goes unrecognized and untreated, these problems become internalized, and can therefore create emotional instability, social withdrawal, and lack of self esteem.

Although it is currently recognized that ADHD is typically a lifelong affliction, one that does not resolve itself even after children enter puberty. In fact, for the majority of children diagnosed with $\mathrm{ADHD}$, as many as $65 \%$, the diagnosis persists into adolescence, ${ }^{2-4}$ however, hyperactivity, although still present, becomes much less visible following childhood. ${ }^{3}$ In the adolescent years, individuals with ADHD may experience greater functional impairment owing to an increased demand on their executive function skills. In some circumstances, this increased demand may expose attentional deficits that were 
not obvious in earlier childhood. ${ }^{5}$ Academic problems that might have been less noticeable or had been treated effectively during elementary school may become much more of a problem in adolescence. ${ }^{6}$ In addition, problems with peer relationships become more obvious given that an individual's social environment changes with the onset of adolescence and that peer interactions assume a new level of importance. A recent study supports these assertions, suggesting that adolescent patients with ADHD could show higher rates of school suspensions and dropouts, teenage pregnancies, and sexually transmitted diseases. ${ }^{7}$

While ADHD is characterized by cognitive and behavioral symptoms of inattention, hyperactivity, and impulsivity, other symptoms as part of comorbid disorders may exist. ADHD is rather than a single homogeneous clinical entity, but a heterogenous group with potentially different etiologic and modifying risk factors and different outcomes. Thus comorbidity is a key clinical feature observed in patients with ADHD throughout the life cycle.

Comorbidity of ADHD with other psychiatric disorders is shown across the entire age span; however, the pattern tends to change according to time of life. For example, comorbid conditions found in children included oppositional defiant disorder $(40 \%)$, conduct disorder (14\%), anxiety disorder (31\%), and mood disorder $(4 \%),{ }^{8}$ whereas adolescence may herald the onset of depression, anxiety, conduct problems, and substance abuse. ${ }^{9,10}$ A study involving 172 ADHD adults found high rates of alcohol abuse or dependence (35\%), oppositional defiant disorder (30\%), conduct disorder (17\%), major depressive disorder (18\%), anxiety disorders (32\%), drug abuse or dependence (14\%) and dysthymia (32\%). ${ }^{11}$ Also adolescents with ADHD are more likely than their non-affected peers to smoke tobacco and cannabis, although rates of alcohol use are not significantly elevated. ${ }^{12}$ Long-term follow-up studies of children diagnosed with ADHD also found that, in late adolescence and early adulthood, there is increased risk for antisocial personality disorder, substance use disorders, and depression. ${ }^{13}$ Biederman also found that comorbidity in adult ADHD patients is similar to that of children, except that antisocial personality replaces oppositional defiant disorder or conduct disorder as the main behavioral psychopathology and mood disorders increase in prevalence. ${ }^{14}$

Even though there has been clinical consensus that the psychiatric comorbidities exhibited by ADHD children may persist, there has been no clear study comparing psychiatric comorbidities between children and adolescents Therefore, we hypothesized that differences may exist in the clinical presentation of ADHD of children and adolescents. To prove this hypothesis, we compared psychiatric comorbid disorders and psychological outcomes in these two groups.

\section{METHODS}

\section{Subjects}

A total of 232 subjects were included in this study. Subjects recruited for the study were children and adolescents who had been referred to outpatient and inpatient clinics at Samsung Medical Center from March 2004 to January 2009. The subjects were divided into two age groups: the child group (aged under 12 years) and the adolescent group (aged 12 years and above). In fact, there are various criteria to define the onset of adolescence. Our criteria was taken from the reference that early adolescence is defined from 12 to 14 years of age and adolescence is classified in three stages, the early, the middle and the late. ${ }^{15}$ All subjects, who were between 5 and 17 years old, were diagnosed with ADHD based on Diagnostic and Statistical Manual of Mental Disorders (DSM) IV diagnostic criteria by a psychiatrist experienced with children and adolescent. Kiddie-Schedule for Affective Disorders and SchizophreniaPresent and Lifetime Korean Version (K-SADS-PL-K) ${ }^{16}$ was performed on all subjects, and for those who were not diagnosed as ADHD were excluded. Three subtypes of ADHD and ADHD Not Otherwise Specified (NOS) had been classified according to the DSM-IV criteria using the K-SADS-PLK. A surveyed point was the same in all subjects, at the first diagnoses. Exclusion criteria included mental retardation, pervasive developmental disorder, psychosis, or significant medical or neurologic illnesses. This study was conducted with the approval of the Institutional Review Board at the Samsung Medical Center.

\section{Comorbidity}

The K-SADS-PL- ${ }^{16}$ is a comprehensive measure of a variety of pathological conditions from both the past and the present, and is useful as a diagnostic interviewing tool for diagnosing major psychiatric disorders in child and adolescent psychiatry. This measure was performed for all subjects as well as their parents in an effort to evaluate psychiatric disorders comorbid with ADHD. All tests were performed by highly trained and supervised psychiatrists.

\section{Psychological outcomes}

We evaluated the intelligence quotient (IQ) of subjects under 16 years old using the Korean Educational Development Institute-Wechsler Intelligence Scale for Children-III and subjects over 16 years old using the Korean-Wechsler Adult Intelligence Scale. In order to examine clinical characteristics, the Korean Version of the Child Behavior Checklist (K-CBCL), which is known to have good validity and reliability, ${ }^{17}$ was used. In fact, the CBCL is one of the most well-studied, empirically derived, checklists available for examining childhood 
psychopathology. ${ }^{18}$ The parents of all patients completed the parents' version of the K-CBCL. ${ }^{17}$

\section{Statistical analysis}

Statistical Analysis System version 9.0 was used for all statistical analysis. Subject characteristics of patients were evaluated using: 1) Chi-Square test or Fisher's Exact test for categorical variables (sex, ADHD subtypes), 2) two-sample t-test or Mann Whitney test for continuous variables (age, IQ). To compare the comorbidity between children and adolescents, chi-square test was conducted and if normality is not satisfied Fisher's exact test was used. Each of ADHD subtype groups were then divided into children and adolescents and then compared psychiatric comorbidity by Fisher's Exact test. To compare psychological outcomes, K-CBCL of children and adolescents were compared. Since there was difference in intelligence quotient between children and adolescents, it has been controlled for comparing K-CBCL. The variables that satisfied normality have taken analysis of covariance and if they failed satisfying normality, Partial Spearman Correlation analysis was con- ducted. On the other hand, for comparing K-CBCL between ADHD subtypes, variables that satisfied normality have taken analysis of variance and if didn't satisfy normality, Kruskal Wallis test was conducted. Each of ADHD subtype groups were then divided into children and adolescents and then compared K-CBCL by Mann Whitney test. A Bonferroni correction was used in a post-hoc analysis. Probability values $<0.05$ were considered significant.

\section{RESULTS}

\section{Subject characteristics}

Of the total, 152 (65\%) patients were children and 80 (34\%) were adolescents. There were 203 (87.5\%) males. No significant gender differences existed between two groups $(\mathrm{p}=0.09$, $\chi^{2}=2.79, \mathrm{df}=1$ ). However, total IQ scores were higher in the child group than the adolescent group ( $\mathrm{p}=0.02$ ). The most numerous among subjects was the combined type, which characterized 110 patients $(47.4 \%)$. The least numerous was the predominantly hyperactive-impulsive type (the hyperactive-

Table 1. Subject characteristics

\begin{tabular}{lccc}
\hline & Children $(\mathrm{N}=152)$ & Adolescents $(\mathrm{N}=80)$ & $\mathrm{p}$ \\
\hline Age, year (mean \pm SD) & $8.25( \pm 1.75)$ & $13.14( \pm 1.27)$ & 0.01 \\
Total IQ (mean \pm SD) & $102.79( \pm 15.89)$ & $97.03( \pm 13.79)$ & 0.02 \\
$\quad$ Verbal IQ & $104.45( \pm 14.81)$ & $95.94( \pm 12.88)$ & 0.02 \\
$\quad$ Performance IQ & $99.96( \pm 16.93)$ & & 0.20 \\
Sex & & $66(82.5 \%)$ & 0.09 \\
Male (\%) & $137(90.1 \%)$ & $14(17.5 \%)$ \\
Female (\%) & $15(9.8 \%)$ & & \\
ADHD subtypes & & $34(42.5 \%)$ & 0.52 \\
Combined (\%) & $76(50.0 \%)$ & $28(35.0 \%)$ \\
Inattentive (\%) & $39(25.7 \%)$ & $2(2.5 \%)$ \\
Hyperactive/Impulsive (\%) & $4(2.6 \%)$ & $16(20.0 \%)$ \\
Not otherwise specified (\%) & $33(21.7 \%)$ & \\
\hline
\end{tabular}

Chi-Square test/Fisher's Exact test with Bonferroni correction, t-test/Mann Whitney test with Bonferroni correction. SD: standard deviation, IQ: intelligent quotient

Table 2. Comorbidity difference between children \& adolescents

\begin{tabular}{lcccr}
\hline & Children $(\mathrm{N}=152)$ & Adolescents $(\mathrm{N}=80)$ & p value & $\chi^{2}$ \\
\hline Conduct disorders & $\mathrm{N}(\%)$ & $\mathrm{N}(\%)$ & $<0.00$ & 1.00 \\
ODD & 0 & $25(8.8)$ & $<0.00$ & 0.33 \\
Mood disorders & $42(27.6)$ & $22(27.5)$ & 1.00 & 16.10 \\
Anxiety disorders & $12(7.9)$ & $16(20.0)$ & 1.00 & 0.00 \\
Tic disorders & $30(19.7)$ & $7(8.8)$ & 0.78 & 0.00 \\
Elimination disorders & $13(8.5)$ & $3(3.8)$ & $<0.01$ & 10.25 \\
Comorbidity found & $11(7.2)$ & $56(70.0)$ & & \\
\hline
\end{tabular}

Chi-Square test/Fisher's Exact test with Bonferroni correction. ODD: oppositional defiant disorder 
impulsive type) for which there were only $6(2.6 \%)$. The predominantly inattentive type (the inattentive type) and NOS characterized 67 (28.9\%) and $49(21.1 \%)$ patients, respectively. And there were no statistical significance between ADHD subtypes $(\mathrm{p}=0.52)$. Detailed information is provided in Table 1 .

\section{Patterns of comorbidity}

Comorbidity differences by age are shown in Table 2 . In the child group, 73 (48.0\%) patients had at least one comorbid disorder as compared to 56 (70.0\%) patients in the adolescent group. The rate of comorbidity in the adolescent group was significantly higher than that for the children $\left(\mathrm{p}<0.01, \chi^{2}=\right.$ $10.25, \mathrm{df}=1$ ). Specifically, the rates of conduct disorder and mood disorder in the adolescent group were significant higher than those in the child group $(\mathrm{p}<0.01)$.
Table 3 represents a comparison of comorbidities in children and adolescents for each of the ADHD subtypes. The adolescent combined type exhibited more conduct disorders than the matched child group ( $\mathrm{p}=0.01)$. Additionally, the adolescent inattentive type demonstrated more mood disorders than the matched child group ( $\mathrm{p}=0.05)$.

\section{Psychological outcomes}

Total IQ scores were higher in the child group than the adolescent group. All subsequent results were adjusted for total IQ scores.

School subscale and total competence subscale scores in the child group were significantly higher than those in the adolescent group (school subscale scores, child group $=46.47$, adolescent group $=43.90, \mathrm{p}=0.009$; /total competence subscale scores,

Table 3. Comparison of comorbidity between children and adolescents in ADHD subtypes and ADHD NOS

\begin{tabular}{|c|c|c|c|c|c|c|c|c|c|}
\hline & \multicolumn{3}{|c|}{ Combined type } & \multicolumn{3}{|c|}{ Inattentive type } & \multicolumn{3}{|c|}{ NOS } \\
\hline & Child. N & Adol. $\mathrm{N}$ & $\mathrm{p}$ & Child. N & Adol. N & $\mathrm{p}$ & Child. N & Adol. N & $\mathrm{P}$ \\
\hline Conduct disorder & 0 & 6 & 0.01 & 0 & 0 & 0 & 0 & 1 & 1.00 \\
\hline ODD & 32 & 16 & 1.00 & 6 & 5 & 1.00 & 4 & 3 & 1.00 \\
\hline Mood disorder & 6 & 7 & 0.84 & 2 & 9 & 0.05 & 4 & 5 & 1.00 \\
\hline Anxiety disorder & 17 & 5 & 1.00 & 9 & 5 & 1.00 & 3 & 5 & 1.00 \\
\hline Tic disorder & 5 & 2 & 1.00 & 3 & 3 & 1.00 & 4 & 2 & 1.00 \\
\hline Elimination disorder & 6 & 0 & 1.00 & 2 & 1 & 1.00 & 2 & 1 & 1.00 \\
\hline
\end{tabular}

Fisher's exact test with Bonferroni correction. ODD: oppositional defiant disorder, Child: children, Adol: adolescents, NOS: not otherwise specified

Table 4. Comparison of K-CBCL between Children and Adolescents in ADHD Subtypes and ADHD NOS

\begin{tabular}{|c|c|c|c|c|c|c|c|c|c|}
\hline & \multicolumn{3}{|c|}{ Combined } & \multicolumn{3}{|c|}{ Inattentive } & \multicolumn{3}{|c|}{ NOS } \\
\hline & $\mathrm{C}$ & A & $\mathrm{p}$ & $\mathrm{C}$ & A & $\mathrm{p}$ & $\mathrm{C}$ & A & $\mathrm{p}$ \\
\hline \multicolumn{10}{|l|}{ Social subscales } \\
\hline Social & 39.4 & 37.6 & 1.00 & 40.3 & 38.4 & 1.00 & 40.7 & 36.2 & 0.37 \\
\hline School & 42.5 & 44.6 & 1.00 & 49.3 & 43.1 & 0.01 & 50.0 & 42.4 & 0.01 \\
\hline Total competence & 36.8 & 37.7 & 0.89 & 42.3 & 36.5 & 0.43 & 42.0 & 35.8 & 0.06 \\
\hline \multicolumn{10}{|l|}{ Clinical subscales } \\
\hline Withdrawn & 58.8 & 56.5 & 0.67 & 59.5 & 55.9 & 0.26 & 59.3 & 55.4 & 0.26 \\
\hline Somatization & 51.3 & 55.5 & 0.22 & 53.2 & 55.9 & 0.98 & 52.4 & 52.0 & 1.00 \\
\hline Anxious/Depressed & 57.4 & 57.7 & 1.00 & 57.0 & 56.2 & 1.00 & 57.9 & 57.1 & 1.00 \\
\hline Social problems & 62.9 & 63.0 & 1.00 & 61.1 & 59.4 & 1.00 & 58.6 & 64.8 & 0.23 \\
\hline Thought problems & 55.9 & 58.2 & 0.98 & 53.9 & 56.6 & 0.59 & 57.0 & 54.0 & 0.35 \\
\hline Attention problems & 63.3 & 64.6 & 1.00 & 59.6 & 61.8 & 0.47 & 59.4 & 62.1 & 1.00 \\
\hline Delinquency & 55.4 & 58.5 & 0.58 & 53.9 & 55.0 & 1.00 & 53.0 & 56.0 & 0.55 \\
\hline Aggression & 61.8 & 63.4 & 1.00 & 55.6 & 55.9 & 1.00 & 58.3 & 55.6 & 1.00 \\
\hline Internalizing problems & 57.8 & 58.1 & 1.00 & 57.6 & 56.2 & 1.00 & 58.0 & 56.1 & 0.92 \\
\hline Externalizing problems & 61.0 & 62.9 & 1.00 & 55.7 & 56.0 & 1.00 & 57.7 & 56.2 & 1.00 \\
\hline Total behavior problems & 60.8 & 62.0 & 1.00 & 57.9 & 58.4 & 1.00 & 58.8 & 57.7 & 1.00 \\
\hline
\end{tabular}

Values are given in mean, Mann Whitney test with bonferroni correction. K-CBCL: Korean-Child Behavior Checklist, C: children, A: adolescents, NOS: not otherwise specified 
child group $=39.74$, adolescent group $=37.15, \mathrm{p}=0.037$, respectively).

When we compared K-CBCL subscales scores between each ADHD subtypes, there were significant differences with respect to aggressive behavior $(\mathrm{p}<0.01)$ and externalizing problems $(p<0.01)$ depending on the ADHD subtypes.

There was no difference found in the K-CBCL scores among the ADHD subtypes in the child group. On the other hand, the adolescent combined type had significantly more aggressive behavior than the adolescent inattentive type (the combined type $=63.4$, the inattentive type $=55.9, \mathrm{p}=0.03$ ).

Table 4 shows a comparison of K-CBCL between children and adolescents in each of the ADHD subtypes. In terms of the inattentive type and NOS, school subscale scores for children were significantly higher than those for adolescents ( $\mathrm{p}<$ 0.01 ). With respect to total competence subscale, children of the NOS demonstrated a trend toward higher scores than the NOS adolescents, although there was no statistical significance. In other types, there were no differences between children and adolescents groups.

\section{DISCUSSION}

In our study, the rate of comorbidity in the adolescent group was significantly higher than that in the child group. In the adolescents group, 56 of 80 subjects (70\%) had at least one comorbid disorder; however, in the child group, the value was 73 of $152(48 \%)$. It is well known that more than $50 \%$ of patients with ADHD have comorbid psychiatric comorbid disorders, ${ }^{19,20}$ and there is even a study which suggested that $79 \%$ of children had at least one comorbid disorder. ${ }^{21}$ However, given that adolescents and children have not been compared to each other with respect to psychiatric comorbidity, the present study may be of significance. Based on our results, it could be suggested that individuals with $\mathrm{ADHD}$ may experience greater functional impairment in adolescence than in childhood. Probably it occurs due to demands for independence, the complexity of social functioning and academic performance that increase in adolescents. Therefore when treating adolescents with $\mathrm{ADHD}$, more careful assessment and treatment targeting a range of comorbidities are needed.

The adolescent group exhibited a significantly higher ratio of comorbid mood disorders than the child group, especially with respect to the inattentive-type. This corresponds to the findings from previous studies reporting that in a sample of 9to 16-year-old subjects, who met the criteria for attention-deficit disorder with or without hyperactivity, $48 \%$ had comorbid depression/dysthymic disorder, 36\% had comorbid oppositional defiant disorder/conduct disorder, and 36\% had comorbid anxiety disorder. ${ }^{22}$ These incidence rates are comparable to the Multisite Multimodal Treatment Study of Children with ADHD (MTA) data for younger children, regarding the comorbidity of anxiety and oppositional defiant disorder with $\mathrm{ADHD}$ but reflect a 12 -fold increase in the reported incidence of depressive/dysthymic symptoms, with an incidence that is much closer to that found for depressive comorbidity in samples of adults with ADHD. ${ }^{23}$ In another 4-year follow-up study, lifetime rates of comorbid depression in children with ADHD also increased from $29 \%$ at baseline to $45 \%$, at an average age of 15 years. ${ }^{24}$

Based on previous and our current results, it is possible that depression has not been properly evaluated in children. Because the reliability of reports from parents and children is lower due to the lack of attention paid by parents to internal symptoms and the great attention paid to external symptoms ${ }^{25-27}$ In this way, parents tend to pay more attention to hyperactivity and impulsive behavior than to emotional difficulties in their children. Additionally, children cannot express their emotions as easily as adolescents, who seem to express feelings of their own emotional discomfort relatively easy. Also, in our study, parents did not report the patients' internalizing problems in K-CBCL. Another explanation could be that the rate of onset of major depression increases with age unlike ADHD, which has the age of onset of less than 7 years old, and maintains a consistent presence over time. ${ }^{28} \mathrm{~A}$ final explanation could be that adolescents with ADHD complain a lot about their depressive mood due to various cumulated negative feedback and functional impairments.

In our study, adolescents of the inattentive type exhibited more comorbid mood disorders. With respect to children of the inattentive type, they have been described by teachers as exhibiting less disruptive behavior but higher degrees of social impairment, unhappiness, and anxiety or depression, as compared to children of the combined type. ${ }^{29}$ Children of the inattentive types have also been found to be more likely to have concurrent internalizing disorders than those of the combined types. ${ }^{30}$ In this regard, children and adolescents seem to have a higher number of mood disorders of the inattentive type than the combined type.

The adolescent group also exhibited a significantly higher ratio of comorbid conduct disorder than the child group. It's been assumed this difference has been resulted due to the fact that none had comorbid conduct disorder in child group, which could imply the age difference between children and adolescents has influenced the diagnosis.

Oppositional defiant disorder was the most common comorbid disorder in both children and adolescence. This finding is in agreement with other recent reports. ${ }^{21,31}$ Studies have shown that $54-84 \%$ of children and adolescents with ADHD may meet the criteria for oppositional defiant disorder. ${ }^{32}$ In the 
present study, the combined type of ADHD children exhibited a significantly higher ratio of comorbid Oppositional Defiant Disorder than did the other types. This is the same finding as other recent reports. ${ }^{33}$ This is also consistent with the results from the K-CBCL, which found that the combined type adolescents had significantly more aggressive behavior.

The second most common comorbid disorder was mood disorder (27.5\%) in adolescents and anxiety disorder (19.7\%) in the child group. Anxiety disorders were the third most common comorbid disorders in adolescents (20.0\%). About one-quarter to one-third of children with ADHD will meet the criteria for an anxiety disorder, compared with $5-15 \%$ of the general population. ${ }^{22,34}$ Additionally, the MTA study found that $34 \%$ of children with ADHD met the criteria of an anxiety disorder. ${ }^{35}$ Even though our results for anxiety disorder have shown lower rates than other studies, the differences between children and adolescents was rather insignificant. This may be because anxiety disorders are sustained for both children and adolescents.

The adolescent group tended to be behind in school subscale and total competence subscale of K-CBCL as compared to the child group. In particular, the inattentive types of adolescents performed significantly lower in school subscale than the inattentive types of children. This could be interpreted as the inattentive types in adolescents only being concerned with attention problems whereas the combined types exhibited various complex problems. Therefore, it may be stated that the inattentive type in adolescents is more distinctive with respect to impairment of academic performance. In addition, the ADHD NOS demonstrated significantly lower school subscale in adolescents versus children. The ADHD NOS has been obscure but is known to be less severe. Regardless, we must consider that there is some clinical dysfunction in the NOS of adolescent ADHD.

Despite its finding, this study has some limitations. First, this study is not a longitudinal follow-up, but rather cross-sectional in nature. If the study would have been followed-up long enough to see children progress into adolescence, more accurate observations with respect to comorbidity and clinical characteristics could have been reported. Therefore, a longitudinal study would be beneficial in further study. Second, the results of this comparison are derived primarily from research on males due to the greater preponderance of males in this study. This may prove to be a confounding factor. Ultimately, comparisons of females and males should have been done, and may prove beneficial in further study. Third, since this study was carried out in only one university hospital, located in a metropolitan area, it is possible that the ADHD patient who visited our hospital had higher incidences of psychopathology than an ADHD group from the general population. There- fore, we should consider that these results may not reflect the general Korean population of children and adolescents.

\section{REFERENCES}

1. Polanczyk G, de Lima MS, Horta BL, Biederman J, Rohde LA. The worldwide prevalence of ADHD: a systematic review and metaregression analysis. Am J Psychiatry 2007;164:942-948.

2. Biederman J, Faraone S, Milberger S, Guite J, Mick E, Chen L, et al. A prospective 4-year follow-up study of attention-deficit hyperactivity and related disorders. Arch Gen Psychiatry 1996;53:437-446.

3. Milich R, Loney J. The role of hyperactivity and aggressive symptomatology in predicting adolescent outcome among hyperactive children. J Pediatr Psychol 1979;4:93-112.

4. Ingram S, Hechtman L, Morgenstern G. Outcome issues in ADHD: adolescent and adult long-term outcome. Ment Retard Dev Disabil Res Rev 1999;5:243-250.

5. Wolraich ML, Wibbelsman CJ, Brown TE, Evans SW, Gotlieb EM, Knight JR, et al. Attention-deficit/hyperactivity disorder among adolescents: a review of the diagnosis, treatment, and clinical implications. Pediatrics 2005;115:1734-1746.

6. Barkley RA, Anastopoulos AD, Guevremont DC, Fletcher KE. Adolescents with ADHD: patterns of behavioral adjustment, academic functioning, and treatment utilization. J Am Acad Child Adolesc Psychiatry 1991;30:752-761.

7. Barkley RA, Fischer M, Smallish L, Fletcher K. Young adult outcome of hyperactive children: adaptive functioning in major life activities. J Am Acad Child Adolesc Psychiatry 2006;45:192-202.

8. Jensen PS, Hinshaw SP, Kraemer HC, Lenora N, Newcorn JH, Abikoff $\mathrm{HB}$, et al. ADHD comorbidity findings from the MTA study: comparing comorbid subgroups. J Am Acad Child Adolesc Psychiatry 2001;40: 147-158.

9. Rucklidge JJ, Tannock R. Psychiatric, psychosocial, and cognitive functioning of female adolescents with ADHD. J Am Acad Child Adolesc Psychiatry 2001;40:530-540.

10. Biederman J. Attention-deficit/hyperactivity disorder: a selective overview. Biol Psychiatry 2005;57:1215-1220.

11. Murphy K, Barkley RA. Attention deficit hyperactivity disorder adults: comorbidities and adaptive impairments. Compr Psychiatry 1996;37: 393-401.

12. Upadhyaya HP, Rose K, Wang W, O'Rourke K, Sullivan B, Deas D, et al. Attention-deficit/hyperactivity disorder, medication treatment, and substance use patterns among adolescents and young adults. J Child Adolesc Psychopharmacol 2005;15:799-809.

13. Fischer M, Barkley RA, Smallish L, Fletcher K. Young adult follow-up of hyperactive children: self-reported psychiatric disorders, comorbidity, and the role of childhood conduct problems and teen CD. J Abnorm Child Psychol 2002;30:463-475.

14. Biederman J, Spencer T, Wilens T. Evidence-based pharmacotherapy for attention-deficit hyperactivity disorder. Int J Neuropsychopharmacol 2004;7:77-97.

15. Pataki CS. Comprehensive Textbook of Psychiatry (9th ed.) vol. 2. In; Sadock BJ, Sadock VA, Ruiz P, editors. Adolescent Development. Philadelphia: Lippincott Williams \& Wilkins, 2009, p.3357

16. Kim YS, Cheon KA, Kim BN, Chang SA, Yoo HJ, Kim JW, et al. The reliability and validity of Kiddie-Schedule for Affective Disorders and Schizophrenia-Present and Lifetime Version-Korean version (K-SADSPL-K). Yonsei Med J 2004;45:81-89.

17. Oh KG, Lee HR. Development of Korean version of Child Behavior Checklist (K-CBCL), Seoul: Korean Research Foundation Report; 1990.

18. Achenbach TM, Ruffle TM. The Child Behavior Checklist and related forms for assessing behavioral/emotional problems and competencies. Pediatr Rev 2000;21:265-271.

19. Spencer T, Biederman J, Wilens T. Attention-deficit/hyperactivity disor- 
der and comorbidity. Pediatr Clin North Am 1999;46:915-927, vii.

20. Pliszka SR. Psychiatric comorbidities in children with attention deficit hyperactivity disorder: implications for management. Paediatr Drugs 2003;5:741-750.

21. Wilens TE, Biederman J, Brown S, Tanguay S, Monuteaux MC, Blake C, et al. Psychiatric comorbidity and functioning in clinically referred preschool children and school-age youths with ADHD. J Am Acad Child Adolesc Psychiatry 2002;41:262-268.

22. Bird HR, Gould MS, Staghezza BM. Patterns of diagnostic comorbidity in a community sample of children aged 9 through 16 years. J Am Acad Child Adolesc Psychiatry 1993;32:361-368.

23. Kessler R. Prevalence of adult ADHD in the United States: results from the National Comorbidity Study Replicaiton (NCS-R). Presented at the 157th annual meeting of the American Psychiatry Association. New York, NY, May 1-6; 2004.

24. Biederman J, Faraone SV, Keenan K, Benjamin J, Krifcher B, Moore C, et al. Further evidence for family-genetic risk factors in attention deficit hyperactivity disorder. Patterns of comorbidity in probands and relatives psychiatrically and pediatrically referred samples. Arch Gen Psychiatry 1992;49:728-738.

25. Piacentini J, Shaffer D, Fisher P, Schwab-Stone M, Davies M, Gioia P. The Diagnostic Interview Schedule for Children-Revised Version (DISC-R): III. Concurrent criterion validity. J Am Acad Child Adolesc Psychiatry 1993;32:658-665.

26. Jensen P, Roper M, Fisher P, Piacentini J, Canino G, Richters J, et al. Testretest reliability of the Diagnostic Interview Schedule for Children (DISC 2.1). Parent, child, and combined algorithms. Arch Gen Psychiatry 1995;52:61-71.
27. Schwab-Stone M, Fisher P, Piacentini J, Shaffer D, Davies M, Briggs M. The Diagnostic Interview Schedule for Children-Revised Version (DISC-R): II. Test-retest reliability. J Am Acad Child Adolesc Psychiatry 1993;32:651-657.

28. Spencer TJ. ADHD and comorbidity in childhood. J Clin Psychiatry 2006;67 Suppl 8:27-31.

29. Carlson CL, Mann M. Sluggish cognitive tempo predicts a different pattern of impairment in the attention deficit hyperactivity disorder, predominantly inattentive type. J Clin Child Adolesc Psychol 2002;31: 123-129.

30. Weiss $\mathrm{M}$, Worling $\mathrm{D}$, Wasdell $\mathrm{M}$. A chart review study of the inattentive and combined types of ADHD. J Atten Disord 2003;7:1-9.

31. Keenan K, Wakschlag LS. More than the terrible twos: the nature and severity of behavior problems in clinic-referred preschool children. J Abnorm Child Psychol 2000;28:33-46.

32. Barkely RA. Attention Deficit Hyperactivity Disorder: A Clinical Handbook, 3rd edition. New York: Guilford; 2005.

33. Elia J, Ambrosini P, Berrettini W. ADHD characteristics: I. Concurrent co-morbidity patterns in children \& adolescents. Child Adolesc Psychiatry Ment Health 2008;2:15.

34. Cohen P, Cohen J, Kasen S, Velez CN, Hartmark C, Johnson J, et al. An epidemiological study of disorders in late childhood and adolescence--I. Age- and gender-specific prevalence. J Child Psychol Psychiatry 1993; 34:851-867.

35. A 14-month randomized clinical trial of treatment strategies for attention-deficit/hyperactivity disorder. The MTA Cooperative Group. Multimodal Treatment Study of Children with ADHD. Arch Gen Psychiatry 1999;56:1073-1086. 